\title{
COMMENTARY
}

\section{Another step in improving the diagnosis of disseminated intravascular coagulation in sepsis}

\author{
Marcel Levi \\ See related research by Gando et al., http://ccforum.com/content/17/3/R111
}

\begin{abstract}
The diagnosis of disseminated intravascular coagulation (DIC) based on composite scoring systems using routinely available coagulation tests has been greatly facilitated. Such scoring instruments not only adequately assess the presence of DIC but also have strong prognostic power for morbidity and mortality. In this issue of Critical Care, Gando and colleagues report on the prospective validation of the Japanese Association of Acute Medicine score for DIC in patients with severe sepsis.
\end{abstract}

Disseminated intravascular coagulation (DIC) is a serious condition with significant morbidity (that is, organ dysfunction due to microvascular thrombosis, thromboembolic complications and profuse bleeding) and is an independent predictor of mortality in critically ill patients. Despite this serious clinical presentation, an adequate diagnosis of DIC is hampered by the limited availability of one simple and reliable test with sufficient diagnostic accuracy. However, combinations of several routinely available coagulation tests may be helpful to establish this diagnosis.

Many years ago, Japanese investigators were the first to develop a DIC scoring system, employing readily available tests such as the platelet count and routine clotting times. Recently this Japanese scoring system was updated by the Japanese Association for Acute Medicine (JAAM). In this issue of Critical Care, Gando and colleagues have prospectively validated this renewed Japanese score in 624 patients with severe sepsis [1]. The authors were able to show the feasibility of the new JAAM score for DIC and its prognostic power for

Correspondence: m.m.levi@amc.uva.nl

Department of Vascular Medicine and Internal Medicine, Academic Medical Center, University of Amsterdam, Meibergdreef 9, 1105 AZ, Amsterdam, the Netherlands multiple organ dysfunction and mortality. In fact, $63 \%$ of nonsurviving patients were classified as having DIC according to the scoring system in comparison with $42 \%$ of sepsis survivors in this study. Interestingly, the investigators were able to convincingly show that persistent DIC was an even worse prognostic indicator for organ dysfunction and mortality compared with patients in whom the DIC rapidly resolved. In conclusion, the renewed JAAM scoring system for DIC seems to be a valuable tool for clinicians to diagnose patients with DIC, which can be helpful to identify patients at high risk and to select patients for supportive treatment options to ameliorate the coagulation status.

It is interesting to compare the utility of this scoring system with other DIC scoring algorithms. In 2001 a simple scoring system was developed by the subcommittee on DIC of the International Society on Thrombosis and Haemostasis (ISTH) [2]. The score can also be calculated based on routinely available laboratory tests; that is, platelet count, prothrombin time, a fibrin-related marker (usually D-dimer), and fibrinogen. A recent study showed that the International Normalization Ratio can be used (instead of prothrombin time prolongation), further facilitating international exchange and standardization [3]. Prospective studies showed that the sensitivity of the DIC score was $93 \%$ and the specificity was $98 \%[4,5]$. Studies in series of patients with specific underlying disorders causing DIC (for example, cancer patients or patients with obstetric complications) show similar results [6,7]. The severity of DIC according to this scoring system was also related to the mortality in patients with sepsis [8].

When comparing the new JAAM scoring system with the ISTH score, it seems the JAAM scoring system was more sensitive than the ISTH score. Interestingly, this higher sensitivity did not lead to a lower specificity, and patients who were negative on the ISTH score but who had DIC according to the new JAAM score had a relatively high mortality. Hence, the JAAM scoring system 
operates at least as well as the ISTH scoring system, although a formal head-to-head comparison is not available at present.

It is tempting to speculate how the diagnosis of DIC may be further improved. DIC is a complication of many disorders that are associated with a systemic inflammatory response [9]. Any form of systemic inflammation will virtually always be associated with activation of coagulation, ranging from changes in molecular markers in coagulation factors with equivocal clinical significance to its most full-blown variant, DIC [10]. Interesting to note is that whereas the hallmark of DIC is activation of coagulation, our diagnostic tests are mostly centered on assays that detect consumption of platelets and coagulation factors. If we want to further improve the diagnosis of DIC, one may hypothesize that tests which will directly assess coagulation activation in vivo will be helpful. Recently, a renewed interest in point-of-care tests to assess an overall picture of coagulation has been raised. Hypercoagulability as demonstrated with thromboelastography was shown to correlate with clinically relevant morbidity and mortality in several studies [11,12], although its superiority over conventional tests has not yet unequivocally been established [13].

In conclusion, the renewed JAAM scoring system for DIC appears to be a valid test for diagnosing DIC in patients with sepsis and may be helpful in specifically tailored management strategies in these patients [14]. A diagnosis of DIC with this new scoring system is a strong predictor for an adverse outcome. Further improvement in the diagnosis of DIC may rely on better tests to assess hypercoagulability, preferably as point-of-care tools.

\section{Abbreviations}

DIC: Disseminated intravascular coagulation; ISTH: International Society on Thrombosis and Haemoastasis; JAAM: Japanese Association for Acute Medicine.

\section{Competing interests}

The author declares that he has no competing interests.

Published: 13 August 2013

\section{References}

1. Gando S, Saitoh S, Ogura H, Fujishima S, Mayumi T, Araki T, Ikeda H, Kotani J, Kushimoto S, Miki Y, Shiraishi S, Suzuki K, Suzuki Y, Takeyama N, Takuma K, Tsuruta R, Yamaguchi Y, Yamashita N, Aikawa N: A multicenter prospective validation study of the Japanese Association for Acute Medicine disseminated intravascular coagulation scoring system in patients with severe sepsis. Crit Care 2013, 17:R111.

2. Taylor FBJ, Toh CH, Hoots WK, Wada H, Levi M: Towards definition, clinical and laboratory criteria, and a scoring system for disseminated intravascular coagulation. Thromb Haemost 2001, 86:1327-1330.

3. Kim HK, Hong $\mathrm{KH}$, Toh $\mathrm{CH}$ : Application of the international normalized ratio in the scoring system for disseminated intravascular coagulation. J Thromb Haemost 2010, 8:1116-1118.

4. Bakhtiari K, Meijers JC, de Jonge E, Levi M: Prospective validation of the international society of thrombosis and haemostasis scoring system for disseminated intravascular coagulation. Crit Care Med 2004, 32:2416-2421.
5. Toh $\mathrm{CH}$, Hoots WK: The scoring system of the Scientific and Standardisation Committee on Disseminated Intravascular Coagulation of the International Society on Thrombosis and Haemostasis: a five year overview. J Thromb Haemost 2007, 5:604-606.

6. Levi M: Disseminated intravascular coagulation in cancer patients. Best Pract Res Clin Haematol 2009, 22:129-136.

7. Thachil J, Toh CH: Disseminated intravascular coagulation in obstetric disorders and its acute haematological management. Blood Rev 2009, 23:167-176.

8. Dhainaut JF, Yan SB, Joyce DE, Pettila V, Basson BR, Brandt JT, Sundin D, Levi $M$ : Treatment effects of drotrecogin alfa (activated) in patients with severe sepsis with or without overt disseminated intravascular coagulation. J Thromb Haemost 2004, 2:1924-1933.

9. Levi $\mathrm{M}$, ten Cate $\mathrm{H}$ : Disseminated intravascular coagulation. N Engl J Med 1999, 341:586-592.

10. Levi M, van der Poll T, Schultz M: New insights into pathways that determine the link between infection and thrombosis. Neth J Med 2012, 70:114-120.

11. Johansson PI, Stensballe J, Vindelov N, Perner A, Espersen K. Hypocoagulability, as evaluated by thrombelastography, at admission to the ICU is associated with increased 30-day mortality. Blood Coagul Fibrinolysis 2010, 21:168-174.

12. Park MS, Martini WZ, Dubick MA, Salinas J, Butenas S, Kheirabadi BS, Pusateri AE, Vos JA, Guymon CH, Wolf SE, Mann KG, Holcomb JB: Thromboelastography as a better indicator of hypercoagulable state after injury than prothrombin time or activated partial thromboplastin time. J Trauma 2009, 67:266-275.

13. Collins PW, Macchiavello LI, Lewis SJ, Macartney NJ, Saayman AG, Luddington R, Baglin T, Findlay GP: Global tests of haemostasis in critically ill patients with severe sepsis syndrome compared to controls. $\mathrm{Br} J$ Haematol 2006, 135:220-227.

14. Tromp M, Tjan DH, van Zanten AR, Gielen-Wijffels SE, Goekoop GJ, van den Boogaard M, Wallenborg CM, Biemond-Moeniralam HS, Pickkers P: The effects of implementation of the Surviving Sepsis Campaign. Neth J Med 2011, 69:292-298.

doi:10.1186/1364-8535-17-448

Cite this article as: Levi: Another step in improving the diagnosis of disseminated intravascular coagulation in sepsis. Critical Care 2013 17:448. 